Eq. (3) and (4) and $n\left(E_{F}\right)$ from specific heat measurements ${ }^{14}$ we find for the WLF model e.g. at $T=30 \mathrm{~K}: \gamma_{\|} \approx-60$. Some experimental evidence of unusually large Grüneisen constants in $\mathrm{V}_{3} \mathrm{Si}$ has been discussed in Ref. ${ }^{2}$. However further experiments are necessary for a quantitative comparison with this theory.

The author wishes to thank Professor P. Fulde and Professor G. Meissner for stimulating discussions.

14 Morin, F. J., Maita, J. P.: Phys. Rev. 129, 1115 (1963).

\title{
W. Dieterich
}

Institut Max von Laue-Paul Langevin

D-8046 Garching bei München

Federal Republic of Germany

\section{Erratum}

\section{R. E. Nettleton "Interaction between Proton Tunneling and Optical Phonons in $\mathrm{KH}_{2} \mathrm{PO}_{4}$ "}

Z. Physik 248, 101 (1971)

In preparation of the above article, several terms were inadvertently omitted in deriving the coupled integral equations which determine the Curie temperature $T_{c}$ and degree of order $\langle Z\rangle_{c}$ at $T_{c}$. When these terms are restored, the transition in the limit $\Gamma \rightarrow 0$, where $\Gamma$ is the tunneling factor, is secondorder, and not first-order as implied by Eqs. $(27 \mathrm{a}, \mathrm{b})$ of the published article. Any conclusions stated therein about the effect of non-rigid proton-photon coupling on the $\Gamma$-dependence of $T_{c}$ are thus rendered uncertain. Conclusions not dependent on Eqs. $(27 \mathrm{a}, \mathrm{b})$ should remain valid.

R. E. Nettleton

University of Kentucky

Department of Physics and Astronomy

Lexington, Kentucky 40506

USA 